\title{
Lactobacillus reuteri Alleviates Gastrointestinal Toxicity of Rituximab by Regulating the Proinflammatory T Cells in vivo
}

\author{
Binyan Zhao', Bailing Zhou', Chunyan Dong, Rui Zhang, Daoyuan Xie, Yaomei Tian and \\ Li Yang *t
}

State Key Laboratory of Biotherapy and Cancer Center, West China Hospital, Collaborative Innovation Center of Biotherapy, Sichuan University, Chengdu, China

OPEN ACCESS

Edited by:

Takahiro Masuda,

Kyushu University, Japan

Reviewed by:

Salam A. Ibrahim,

North Carolina Agricultural

and Technical State University,

United States

Minako Ito

Kyushu University, Japan

*Correspondence:

Li Yang

yl.tracy73@gmail.com

${ }^{\dagger}$ These authors have contributed equally to this work

Specialty section: This article was submitted to

Microbial Immunology,

a section of the journal

Frontiers in Microbiology

Received: 23 December 2020 Accepted: 27 August 2021

Published: 12 October 2021

Citation:

Zhao B, Zhou B, Dong C, Zhang R, Xie D, Tian $Y$ and Yang $L$ (2021) Lactobacillus reuteri Alleviates Gastrointestinal Toxicity of Rituximab by Regulating the Proinflammatory $T$

Cells in vivo.

Front. Microbiol. 12:645500 doi: 10.3389/fmicb.2021.645500
Rituximab (RTX) is a widely used anticancer drug with gastrointestinal side effects, such as nausea, vomiting, and diarrhea. The reason for these side effects is still poorly understood. Previous studies have reported that the intestinal microbiota is associated with the occurrence of disease and the therapeutic effect of drugs. In this study, we observed mucosal damage, inflammatory cell infiltration and increased intestinal inflammatory factor expression in RTX-treated mice. RTX also changed the diversity of the intestinal microbiota in mice, and decreased abundance of Lactobacillus reuteri was observed in RTX-treated mice. Further experiments revealed that intragastric administration of $L$. reuteri in RTX-treated mice attenuated the intestinal inflammatory response induced by RTX and regulated the proportion of helper T (Th) cells. In conclusion, our data characterize the effect of the intestinal microbiota on RTX-induced intestinal inflammation, suggesting that modifying the gut microbiota may represent a positive strategy for managing adverse reactions.

Keywords: rituximab, intestinal microbiota, Lactobacillus reuteri, gavage methods, gastrointestinal toxicity

\section{INTRODUCTION}

Although there are many new methods for cancer treatment, drug therapy for cancer is still an important and indispensable treatment modality that is mainly based on the ability of drugs to clear cancer cells, reduce tumor growth and relieve pain. Anticancer drugs kill tumor cells by specifically recognizing surface markers on tumor cells and stimulating the immune response. For example, rituximab binds to the $\mathrm{CD} 20$ antigen on B-cell-derived tumor cells to initiate an immune response that mediates B-cell lysis. Possible mechanisms of B-cell lysis include complementdependent cytotoxicity (CDC) and antibody-dependent cytotoxic (ADCC) effects. These killing effects are not specific to tumor cells and also affect $\mathrm{B}$ cells, T cells, and regulatory $\mathrm{T}$ cells. Infection and gastrointestinal toxicity often occur during the course of treatment (El Fassi et al., 2008; Agathocleous et al., 2010; Ardelean et al., 2010; Blombery et al., 2011; Sekkach et al., 2011; Lipka et al., 2016; Shahmohammadi et al., 2018; Kaegi et al., 2019), and they can treatment efficacy and 
patient compliance to a certain extent. However, there is currently no effective treatment to reduce gastrointestinal mucosal lesions. After proliferation and differentiation, crypt cells migrate to the villi of the small intestine to replace the exfoliated mature epithelial cells and become intestinal mucosal epithelial cells (Farrell et al., 1998; Bau et al., 2016). Some drugs kill crypt stem cells of the small intestine, damaging the absorption and barrier function of the intestinal mucosa and resulting in gastrointestinal tract toxicity and side effects. Some studies have shown that in cancer chemotherapy, the incidence of intestinal mucositis is as high as 40\% (Boukhettala et al., 2009; Beutheu et al., 2014). An increasing number of studies have shown that the intestinal microbiota is involved in regulating the efficacy and toxicity of chemotherapy drugs (Li et al., 2016, 2017; Wardill and Tissing, 2017; Wilson and Nicholson, 2017; Zimmermann et al., 2019).

There are a large number of microbial communities in the intestine, including approximately $3 \times 10^{13}$ bacteria (Sender et al., 2016). Most of them coexist with the host in a dynamic balance. However, when the health status of the host is affected, the composition of intestinal flora and the abundance of certain bacteria change. Expansion of some symbiotic bacteria may lead to more serious effects, while the reduction of some symbiotic bacteria will cause the opposite effects; for example, Routy et al. (2018) found that patients with high Akkermansia muciniphila abundance tend to experience improved efficacy after receiving PD-1 therapy (Zhou et al., 2018). Intestinal microbes even play an important role in the health of the host by regulating the immune response or the well-known "liver-brain-gut" axis circulation. When the tissue is damaged after infection or injury, inflammatory cells enter the intestine from the systemic circulation or intestinal cistern to restore the body's balance (Zhou et al., 2018). Therefore, intestinal injury caused by RTX may cause an imbalance in enteric cells, which may lead to malignant inflammatory circulation.

It should be noted that a large number of inflammatory cells in the intestine can also affect the intestinal microbiota. In addition, some drugs can affect the intestinal microbiota. For example, Viaud et al. found that after 7 days of cyclophosphamide treatment, the total number of bacteria in the small intestine of mice did not decrease, but the abundance of Lactobacillus and enterococci decreased. Moreover, after drug treatment, the villi of the small intestine in mice were shortened, the intestinal barrier was damaged, and inflammatory cells had aggregated (Viaud et al., 2013). Moreover, specific gram-positive bacteria (Lactobacillus johnsonii and Enterococcus hirae) regulate the accumulation of cyclophosphamide-driven Th1 and Th17 cell responses (Viaud et al., 2013). It has been reported that CTLA4 antagonists induce $\mathrm{T}$ cell-mediated intestinal mucositis, which is related to changes in the intestinal flora (Viaud et al., 2013; Authors et al., 2015; Sivan et al., 2015; Vétizou et al., 2015). These studies demonstrate that homeostasis of the intestinal flora is important for the host immune system and intestinal epithelium. In our study, we identified the effect of rituximab on intestinal mucosal injury, the changes in different immune cells in mesenteric lymph nodes, and the resulting changes in intestinal microbiota, which are associated with the development of intestinal mucositis. We also found that this toxicity is attenuated by intragastric administration of specific Lactobacillus species. Our aim was to characterize these changes and examine the effects of altering the intestinal microbiota on rituximabinduced mucositis. These findings may contribute to subsequent studies on methods to attenuate the gastrointestinal toxicity of certain drugs.

\section{MATERIALS AND METHODS}

\section{Animal Experiments}

Female BALB/c mice (6-8 weeks old) were purchased from Beijing Vital River Laboratory Animal Co., Ltd. (Beijing, China). All animals were housed in a pathogen-free environment and maintained at $22 \pm 2{ }^{\circ} \mathrm{C}$ under 12 -h light/dark cycle conditions. All animal experiments were performed according to the guidelines approved by the Animal Protection Committee of Sichuan University (Sichuan, China, ID: 2018091815).

Two experiments were performed to investigate the relationship between rituximab toxicity and the intestinal microbiota. In the first experiment, female $\mathrm{BALB} / \mathrm{c}$ mice were divided into two groups. Mice in the experimental group were intraperitoneally injected with $4 \mathrm{mg}$ rituximab (Roche, $100 \mathrm{mg} / 10 \mathrm{ml}$ ), while mice in the control group received sterile phosphate balanced solution (PBS). In the second experiment, female BALB/c mice were randomly divided into six groups with six mice per group. Mice in the antibiotic (ATB) group, $\mathrm{ATB}+\mathrm{RTX}$ group and $\mathrm{ATB}+\mathrm{RTX}+$ Lactobacillus reuteri group were treated with metronidazole (1 g/L, Solarbio 44348-41) supplied in the drinking water on day -7 for 5 days that was then stopped for 2 days. On day 0, mice in the RTX and ATB + RTX groups were intraperitoneally injected with rituximab at $4 \mathrm{mg} /$ mouse. Mice in the RTX + L. reuteri and $\mathrm{ATB}+\mathrm{RTX}+$ L. reuteri groups were simultaneously gavaged with $1 \times 10^{8} \mathrm{CFU}$ of $L$. reuteri in $200 \mu \mathrm{l}$ PBS for 7 days. The other two groups (ATB and Con) were gavaged with sterile PBS. All mice were sacrificed on day 7 .

\section{Bacterial Culture}

Lactobacillus reuteri (ATCC 53608) was purchased from American Type Culture Collection (ATCC) and were cultured in De Man, Rogosa and Sharpe (MRS) broth medium (M264$02)$ at $37^{\circ} \mathrm{C}$ and under anaerobic conditions. The bacterial suspension was washed with sterile PBS, centrifuged $(3,200 g$, $5 \mathrm{~min}$ ) and resuspended in PBS until $\mathrm{OD}_{600}=1$, which was approximately $1 \times 10^{8}$ colony forming units (CFUs)/ml (Jiménez-Flores et al., 2010).

\section{Stool Sample Collection and DNA Extraction}

According to different animal experimental schemes, stool samples were collected from mice on days $-7,0$, and 7 during the first experiment and immediately stored at $-80^{\circ} \mathrm{C}$. In the second animal experiment, fecal samples were collected from mice on days $-7,-5,0$, and 7 and were immediately stored at $-80^{\circ} \mathrm{C}$ until processing with a fecal DNA isolation kit (Foregene Co., 
Ltd., Sichuan, China). Samples were stored at $-80^{\circ} \mathrm{C}$ according to the fecal DNA isolation kit manufacturer's instructions.

\section{S Sequencing and Bioinformatics Analysis}

The V4 region of $16 \mathrm{~S}$ rDNA was amplified by Beijing Novogene Technology Co., Ltd., and sequenced on the NovaSeq6000 platform. Paired-end reads were merged using FLASH (V1.2.7) ${ }^{1}$ (Magoč and Salzberg, 2011), a fast and accurate analysis tool that was designed to merge paired-end reads when at least some of the reads overlapped reads generated from the opposite end of the same DNA fragment, and the splicing sequences were represented raw tags. Quality filtering of the raw tags was performed under specific filtering conditions to obtain high-quality clean tags (Bokulich et al., 2013) according to QIIME (V1.9.1). ${ }^{2}$ Tags were subsequently compared with the reference database (Silva database) ${ }^{3}$ using the UCHIME algorithm (UCHIME algorithm) ${ }^{4}$ (Edgar et al., 2011) to detect chimeric sequences, which were then removed (Haas et al., 2011), yielding the effective tags.

Sequence analysis was performed using Uparse software (Uparse v7.0.1001) $)^{5}$ (Edgar, 2013). Sequences with $\geq 97 \%$ similarity were assigned to the same OTUs.

Representative sequences for each OTU were screened for further annotation. For each representative sequence, the Silva Database (see text footnote 3) (Quast et al., 2013) was used based on the Mothur algorithm to annotate taxonomic information. To study the phylogenetic relationship of different OTUs and the difference in the dominant species in different samples (groups), multiple sequence alignment was conducted using MUSCLE software (Version 3.8.31) ${ }^{6}$ (Edgar, 2004).

Linear discriminant analysis (LDA) and effect size (LEfSe) analysis methods (Segata et al., 2011) used the Kruskal-Wallis test to compare the abundance of all bacterial clades between day 0 and day 7 at a predefined $\alpha$ of 0.05 . Significantly different vectors obtained by comparing abundance among the groups were used as inputs for LDA, resulting in an effect size. Compared with traditional statistical tests, the main advantage of LEfSe is that in addition to the $p$-value, LEfSe also produces an effect size. This feature allows us to sort the results of multiple tests based on the size of the difference between groups.

\section{Quantitative Real-Time PCR}

Quantitative real-time PCR (qPCR) was used to quantify bacterial $16 \mathrm{~S}$ rDNA gene abundance in feces samples. Primers targeting the rDNA gene of L. reuteri (F: ACCGAGAACACCGCGTTATTT, R: CATAACTTAACCTAAACAATCAAAGATTGTCT) (Rothhammer et al., 2016) and primers targeting $\beta$-actin (F: CCCAGGCATTGCTGACAGG, R: TGGAAGGTGGACAGTGA GGC) were used.

\footnotetext{
${ }^{1}$ http://ccb.jhu.edu/software/FLASH/

${ }^{2} \mathrm{http}: / /$ qiime.org/scripts/split_libraries_fastq.html

${ }^{3}$ https://www.arb-silva.de/

${ }^{4} \mathrm{http}: / /$ www.drive5.com/usearch/manual/uchime_algo.html

${ }^{5} \mathrm{http}: / /$ drive5.com/uparse/

${ }^{6} \mathrm{http}: / / \mathrm{www}$. drive5.com/muscle/
}

Total RNA was extracted from cells or small intestine segments of mice using a kit (Foregene, China) and was quantified using a Nanodrop ND-1000 spectrophotometer (Thermo Scientific). According to the manufacturer's instructions, cDNA was synthesized using a PrimeScript RT Kit with gDNA Eraser (Vazyme, China). Transcription levels of TNF- $\alpha$, IL-1 $\beta$, IL-6, IL-10, claudin-1, and $\beta$-actin were analysed by SYBR Green PCR Master Mix (Vazyme, China) on a CFX96 real-time system (BioRad). The relative expression was calculated by $\Delta C T$ method. The sequences of the gene-specific primers were as follows: TNF- $\alpha$ (F: CCCAGGGACCTCTCTCTAATC, R: ATGGGCTACAGGCTTGTCACT); IL-1 $\beta$ (F: ATCTCGCAGCA GCACATCAA, R: ACGGGAAAGACACAGGTAGC); IL-6 (F: CCAGTTGCCTTCTTGGGACT, R: GTCTCCTCTCCGGACTT GTG); IL-10 (F: CATCGATTTCTTCCCTGTGAA, R: TCTTGGAGCTTATTAAAGGCATTC); and claudin-1 (F: TGCCCCAGTGGAAGATTTACT, R: CTTTGCGAAACG CAGGACAT).

Quantitative real-time PCR was performed on a CFX96 Realtime system (Bio-Rad) under the following cycling conditions: $95^{\circ} \mathrm{C}$ for $30 \mathrm{~s}, 35$ cycles at $95^{\circ} \mathrm{C}$ for $10 \mathrm{~s}$, and $60^{\circ} \mathrm{C}$ for $30 \mathrm{~s}$. Then, the default melting curve acquisition program was used.

\section{Hematoxylin and Eosin Staining for Histological Analyses}

The mice were sacrificed 1 week after RTX treatment. Ileum and colon samples were fixed in 4\% PFA for at least $48 \mathrm{~h}$. Subsequently, the tissues were cut into small pieces, embedded in paraffin and cut into $4-\mu \mathrm{m}$ sections that were stained with hematoxylin and eosin (H\&E) and scored by pathologists. In the semi-quantitative analysis of histology, villus atrophy, necrosis, and inflammatory cell infiltration scores were as follows: 0 (no injury), 1 (mild injury), 2 (moderate injury), and 3 (severe injury). The lengths of 10 intact and well-oriented villi were measured using ImageJ software (Yu et al., 2019).

\section{Flow Cytometry Experiments}

Monoclonal antibodies against mouse CD3E, CD4, Foxp3, IL-4, CD19, IL-10, IFN- $\gamma$, IL-17A, CD11b, CD11c, F4/80, CD206, and CD16/32 were purchased from BD Biosciences. All mice were sacrificed at the end of the experiment. Mesenteric lymph nodes (MLNs) and spleens were crushed in a cell filter (75 $\mu \mathrm{m}$ nylon; $\mathrm{BD}$ Falcon), and the cell suspension was treated with erythrolysis buffer (Beyotime Biotechnology) at room temperature for $5 \mathrm{~min}$. Then, the cells were washed three times and suspended in PBS. For surface antibody staining, the cell suspension was combined with various antibodies and incubated in the dark at $4^{\circ} \mathrm{C}$ for 30 min. To stain for Foxp3, IL-4, IL-10, IL-17A, and IFN- $\gamma$, the cells were fixed in $4 \%$ neutral paraformaldehyde after surface marker staining, treated with $0.5 \%$ Triton-100 (PBS dilution) at $4^{\circ} \mathrm{C}$ for $20 \mathrm{~min}$, washed again, and then incubated with corresponding antibodies. Flow cytometry (FACSCalibur; BD Biosciences) was used to obtain the cell events, and the data were analysed with NovoExpress software. 


\section{Cell Culture and Stimulation With Lactobacillus reuteri in vitro}

The mesenteric lymph node (MLN) cells of mice were cultured at $37^{\circ} \mathrm{C}$ and $5 \% \mathrm{CO}_{2}$. Ten percent fetal bovine serum (Gibco, United States) was added to RPMI 1640 cell culture medium. For L. reuteri's anti-inflammatory test, MLN cells were cultured in a 6-well plate at a density of $2 \times 10^{6}$ cells per well and then coincubated with $L$. reuteri (infection multiple $=10,2 \times 10^{6}$ cells and $\left.2 \times 10^{7} \mathrm{CFU}\right)$ in the presence or absence of LPS $(10 \mathrm{ng} / \mathrm{mL}$, PrimeGene) (Chae, 2018) for $4 \mathrm{~h}$. After incubation, cells were collected for gene expression analysis.

\section{Statistical Analysis}

All data were statistically analysed using GraphPad Prism 8 and are shown as the mean value \pm SEM of the mean. Statistical significance was evaluated at a level of $P<0.05$ using unpaired two-way analysis of variance (ANOVA) or $t$-test analysis, * indicated significance at $p<0.05,{ }^{* *} p<0.01,{ }^{* * *} p<0.001$, **** $P<0.0001$, respectively.

\section{RESULTS}

\section{Rituximab Induces Intestinal Mucositis in Mice}

To investigate the intestinal toxicity of RTX, mice were treated with a single dose of RTX (4 mg/mouse, i.p.) for 7 days (Figure 1A). Compared with the control group, RTX caused significant weight loss in mice (Figure 1B). In contrast to the control group, villous atrophy and intestinal epithelial injury of mice in the RTX treatment group were obvious, as shown in Figure 1C. Moreover, obvious inflammatory cell infiltration was apparent in the colon tissue of RTX-treated mice (Figure 1C). Compared with the control group, the intestinal mucositis score (Supplementary Figure 2) in the RTX group
A

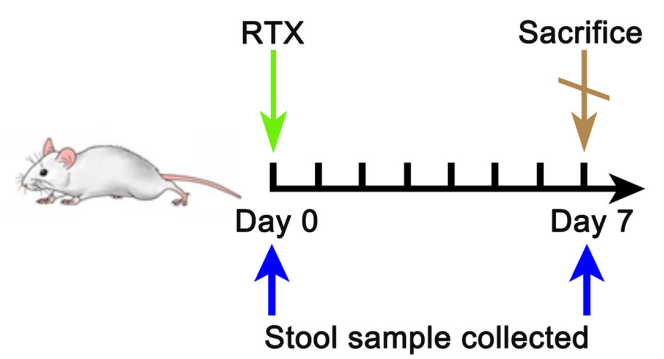

C

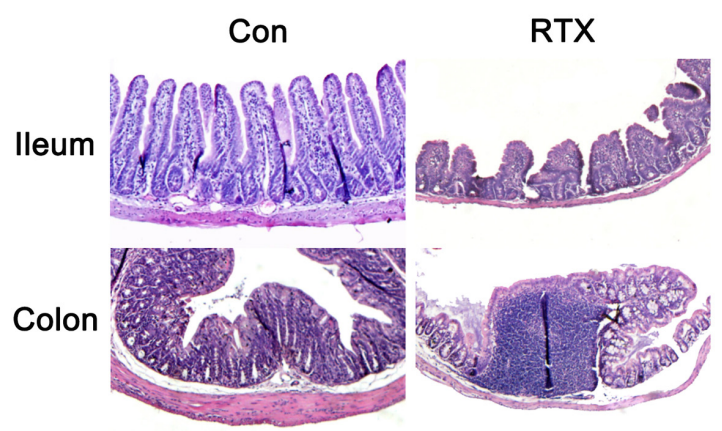

B

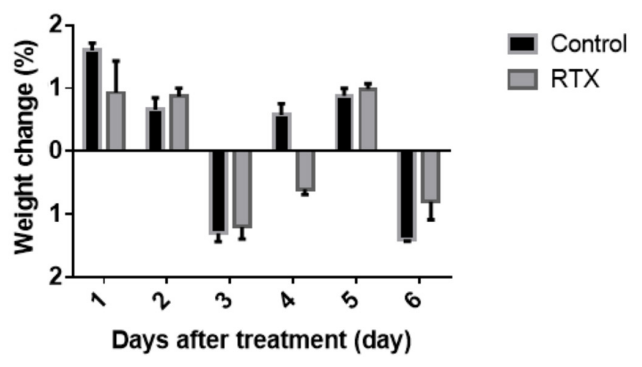

D

E
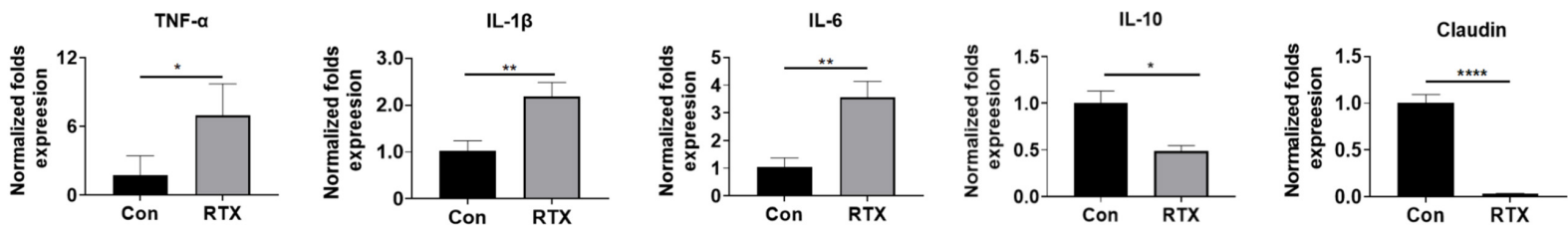

FIGURE 1 | Rituximab (RTX) causes marked intestinal mucositis. (A) Schematic diagram of RTX administration and stool sample collection. BALB/c mice were intraperitoneally injected with RTX or normal saline on day 0 and then sacrificed on day 7 . Feces were collected before and after treatment. (B) From day 0 to day 7 , the body weights of mice in the control and RTX treatment groups were changed. (C) H\&E staining of the ileum and colon (magnification, 40x). (D) Villus length of ileum and colon. (E) Expression levels of TNF- $\alpha$, IL-1 $\beta$, IL-6, IL-10, and claudin in the ileum of mice treated with RTX or normal saline. Data are expressed as the mean \pm SEM. ${ }^{*} p<0.05,{ }^{* *} p<0.01,{ }^{* \star \star *} P<0.0001$. 
was significantly increased $(P<0.001$; Figure 1D). These results suggest that RTX treatment causes intestinal damage and inflammatory cell infiltration. To further verify the intestinal inflammatory response induced by RTX, qPCR was used to assess the expression of inflammatory markers in the ileum. We observed that expression levels of the proinflammatory cytokines TNF- $\alpha$, IL- $1 \beta$, and IL- 6 in the ileum of RTX-treated mice were significantly increased, while expression of IL-10 was significantly decreased (Figure 1E), the colon inflammatory factor data in the Supplementary Figure 3. Claudin-1 is a tight junction transmembrane protein that constitutes tight junctions between cells. It functions mainly to regulate the permeability of the barrier structure. The stability of tight junctions is related to the complex interactions between claudin proteins and other tight junction proteins. Our results showed that compared with the control group, the expression of claudin in the intestinal tissue of RTX-treated mice was significantly decreased $(P<0.0001$; Figure 1E), which may increase intestinal permeability, cause bacterial migration to the site of intestinal injury, and further promote the local inflammatory response.

\section{Rituximab Induces Alterations in T Cell Subsets}

We observed that compared with the control group, the infiltration of $\mathrm{CD}^{+}$dendritic cells in intestinal tissues were significantly increased after RTX treatment (Supplementary Figure 1), and $\mathrm{CD}^{+}$cells were subdivided into subsets of $\mathrm{T}$ helper (Th), such as Th1, Th2, Th9, Th17, regulatory T (Treg) cell, and follicular helper $\mathrm{T}$ cell subtypes. Our results indicated that compared with the control group, levels of Th1 (CD4 ${ }^{+} / \mathrm{IFN}-$ $\left.\gamma^{+}\right)$and Th17 cells $\left(\mathrm{CD} 4^{+} / \mathrm{IL}_{-17 \mathrm{~A}^{+}}\right)$in the MLN of RTXtreated mice were significantly increased (Figure 2 ). Th1 cells promote the cell-mediated inflammatory response by inducing the activation of macrophages, NK cells, and B cells (Carrasco and Fernández-Bañares, 2017). Most of the effector capacity of Th17 cells comes from the fact that IL-17, together with TNF$\alpha$, strongly promote inflammation by inducing the expression of adhesion molecules, proinflammatory cytokines (e.g., IL-6, GM-CSF, and G-CSF), chemokines, prostaglandin E2 and matrix metalloproteinases (Kumawat et al., 2013; Rauber et al., 2017). Th17 cells respond to infection by extracellular bacteria and fungi. Elevated Th17 cells have been found in patients with atopic dermatitis, Crohn's disease, psoriasis, and multiple sclerosis.

In addition, macrophages can adopt specialized functional phenotypes in response to various signals, known as classic activated macrophages $\left(\mathrm{CD} 11 \mathrm{~b}^{+} \mathrm{F} 4 / 80^{+}\right)$and selectively activated macrophages $\left(\mathrm{CD} 11 \mathrm{~b}^{+} \mathrm{CD} 206^{+}\right)$(Mantovani et al., 2002; Sica and Mantovani, 2012). CD11b ${ }^{+} \mathrm{F} 4 / 80^{+}$macrophages secrete proinflammatory cytokines, present antigens, promote the Th1 response, and mainly participate in the positive immune response. In contrast, $\mathrm{CD} 11 \mathrm{~b}^{+} \mathrm{cd} 206^{+}$macrophages play an immunomodulatory role by producing inhibitory cytokines, such as IL-10 or TGF- $\beta$, and downregulating the immune response (Sica and Bronte, 2007; Martinez and Gordon, 2014; Acharya et al., 2020). Compared with the control group, the content of $\mathrm{CD} 11 \mathrm{~b}^{+} \mathrm{F} 4 / 80^{+}$macrophages in the spleen and MLN were increased after RTX treatment, while $\mathrm{CD} 11 \mathrm{~b}^{+} \mathrm{CD} 206^{+}$ macrophages were slightly decreased (Supplementary Figure 1). In addition, the ratio of $\mathrm{CD}_{11 \mathrm{~b}}{ }^{+} \mathrm{F} 4 / 80^{+} / \mathrm{CD} 11 \mathrm{~b}^{+} \mathrm{CD} 206^{+}$ cells in the RTX treatment group was significantly increased, suggesting that RTX promotes the polarization of inflammatory macrophages induced by $\mathrm{CD} 11 \mathrm{~b}^{+} \mathrm{F} 4 / 80^{+}$cells in mice, inducing intestinal inflammation (Supplementary Figure 1).

\section{Rituximab Induces Changes in Intestinal Microbial Components}

Recent studies have shown that some drugs cause changes in the intestinal microbial composition, such as cyclophosphamide and carboplatin. In addition, the intestinal microbiota is closely related to drug resistance, efficacy, and toxicity (Alexander et al., 2017; Bullman et al., 2017; Gopalakrishnan et al., 2018; Matson et al., 2018; Routy et al., 2018; Zhou et al., 2018). To determine whether RTX has an effect on the intestinal flora of mice, 16S ribosomal DNA (rDNA) sequencing was used, collecting stool samples before and after RTX treatment for bacterial microbiota profiling. RTX treatment changed the microbial population in mice (Figure 3A). Although most OTUs were shared on day 0 and day 7, 153 OTUs were lost, 105 unique OTUs appeared after RTX treatment, and the total number of OTUs decreased. Next, we analysed the bacterial alpha diversity in the RTX treatment and control groups. We found that the total microbial diversity decreased significantly in the RTX treatment group, while no significant change was observed in the control group over time (Figures 3B,C). Moreover, principal component analysis (PCA) revealed that the composition of the intestinal microflora changed significantly after RTX treatment (day 7) compared with before RTX treatment (day 0) (Figure 3D). In the control group, there was little difference in intestinal microbiota composition between days 7 and 0 (Figure 3D). In addition, significant changes in the intestinal microbiota were estimated by comparing the composition of OTUs with that of normal mice. Figure $3 \mathrm{E}$ shows the increase or decrease in microbial components in response to different treatments. For example, the relative abundance of Lactobacillus decreased significantly (Figure 3F), while there was no significant change in these OTUs between day 0 and day 7 in the control group. In conclusion, these data suggest that RTX treatment alters the diversity and composition of the intestinal microbiota in mice (the comparison of RTX and control groups about the results of $\alpha$ and $\beta$ diversity on day 0 and day 7 was shown in Supplementary Figure 4).

\section{Identification of Key Microbial Species}

To identify the most differentially abundant bacteria after RTX treatment, LDA effect size analysis (LEfSe) was performed, and we selected biomarkers with LDA scores $>4$. We found that Lactobacillus was more abundant before RTX administration and decreased after 7 days (Figures 4A,B). We further analysed the relative abundance of different Lactobacillus species in the control and RTX treatment groups. The results showed that the most differentially abundant bacteria were Lactobacillus gasseri (L. gasseri) in the RTX treatment group, consistent with the 


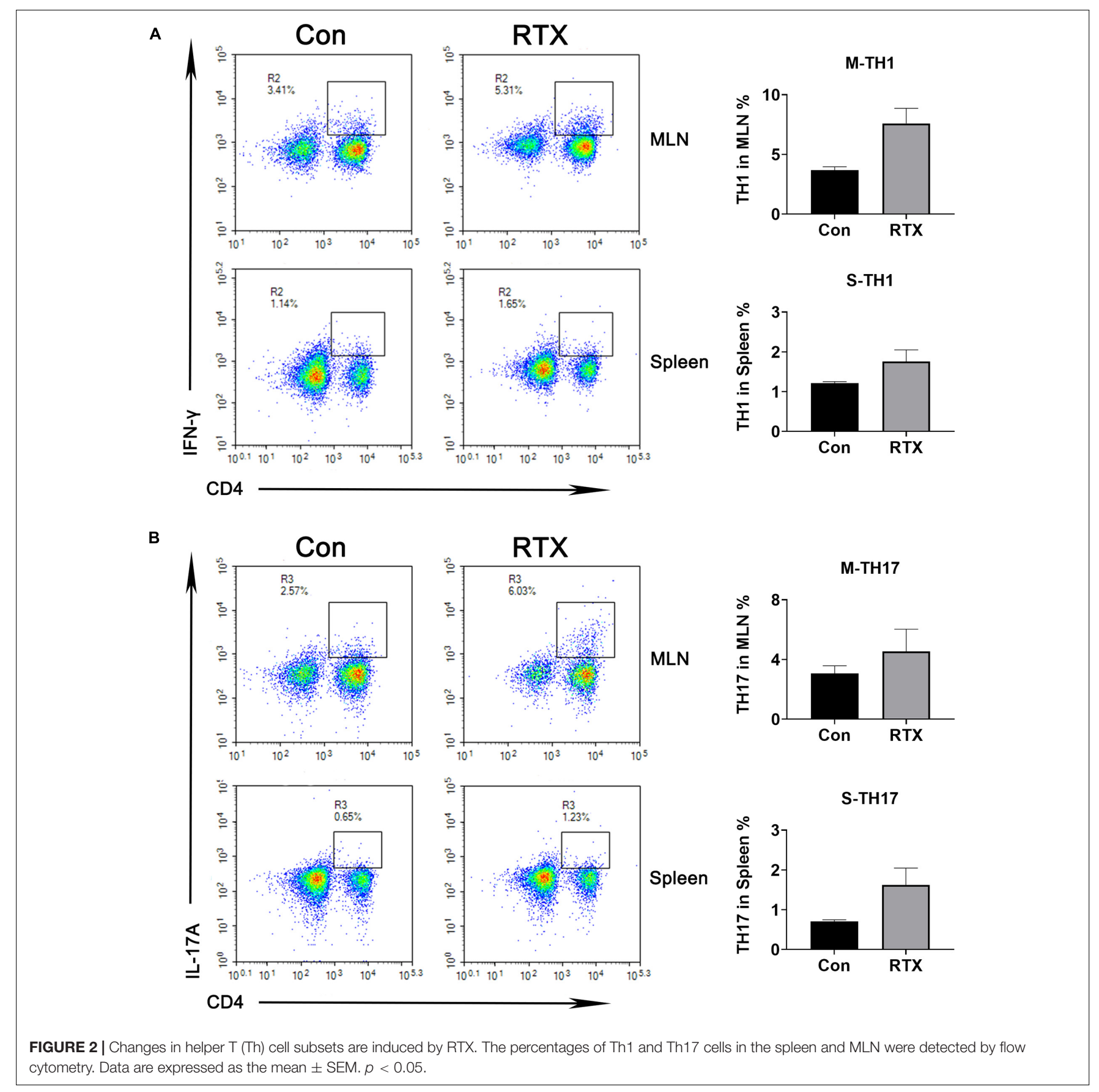

control group (Figure 4C). Only L. reuteri decreased significantly in the RTX-treated group, while no significant change was observed in the control group. These results demonstrate that the number of $L$. reuteri decreased significantly after RTX treatment compared with the control group. L. reuteri exhibits strong adhesion to the intestinal mucosa. It improves the distribution of intestinal microbiota, inhibits the colonization of harmful bacteria and resists intestinal diseases. We conducted qPCR to quantify the number of specific Lactobacillus in feces samples. After RTX treatment, the amount of L. gasseri decreased, but the number in the control group also decreased, while the number of $L$. reuteri did not decrease after PBS treatment (Figure 4D), consistent with previous reports. It should be noted that the changes in L. reuteri after RTX treatment were consistent with the $16 \mathrm{~S}$ rDNA sequencing results, suggesting that L. reuteri may play a role in RTX-induced intestinal mucositis.

\section{Lactobacillus reuteri Inhibits Inflammation in vitro}

To invaluated the role of $L$. reuteri in RTX-induced intestinal mucositis. Next, we evaluated whether $L$. reuteri inhibits 
A

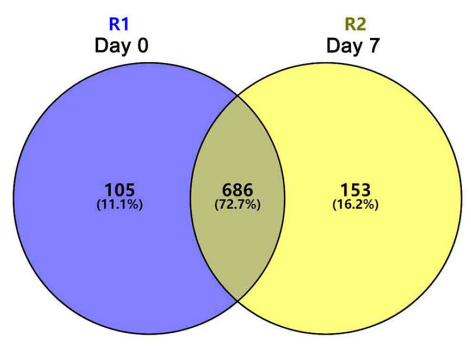

C

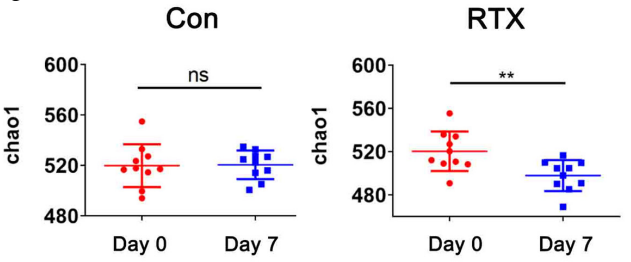

B

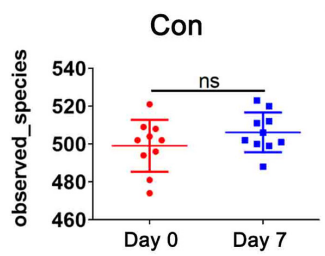

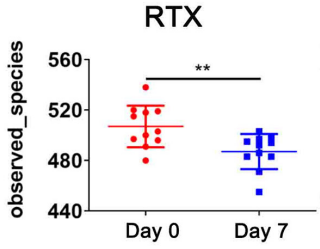
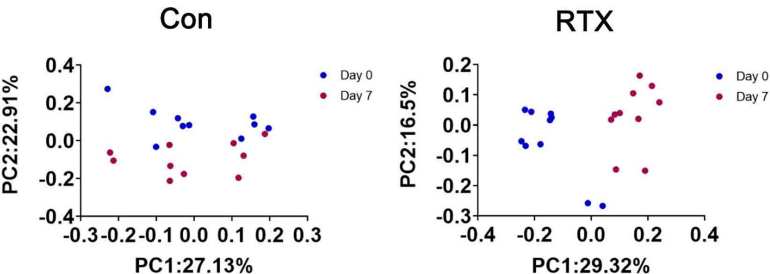

$\mathbf{E}$
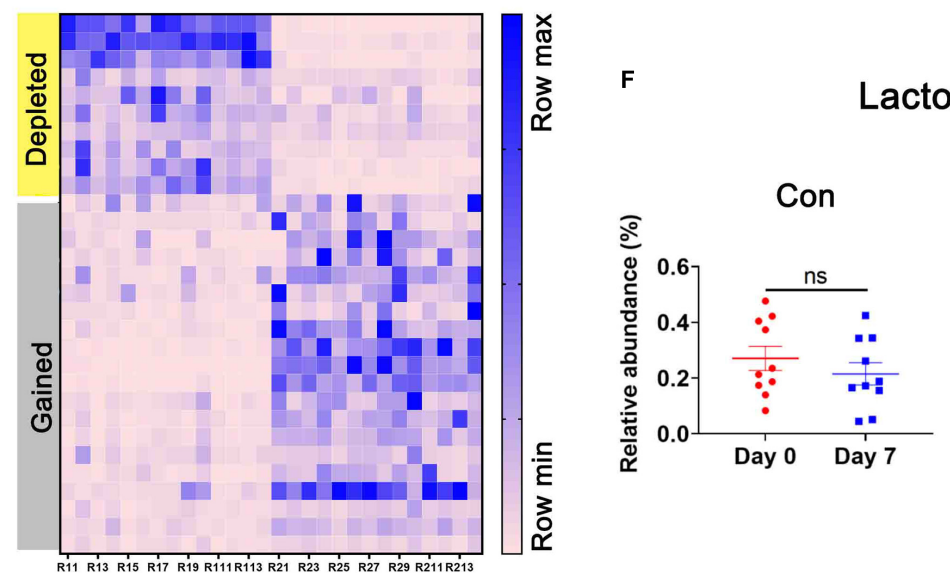

Lactobacillus

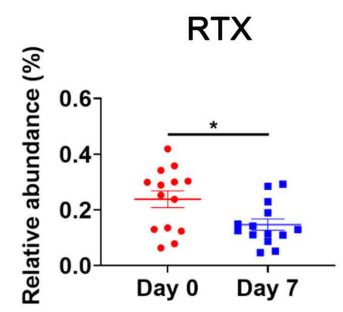

FIGURE 3 | Rituximab treatment changes the composition of the intestinal microbiota. (A) The number of OTUs in the intestine of RTX-treated mice on day 0 (blue), day 7 (yellow), or shared on days 0 and 7 (gray). (B,C) Changes in the $\alpha$ diversity of the intestinal microbiota in the RTX-treated and control groups are shown on days 0 and 7. (D) The results of principal component analysis (PCA) of intestinal microbiota in the RTX-treated group and control group on days 0 and 7 .

(E) Heatmap of OTUs of intestinal microbiota in RTX-treated mice on days 0 and 7. (F) The relative abundance of 2 examples of OTUs in RTX-treated mice and control mice. OTUs, operational taxonomic units. ${ }^{\star} p<0.05,{ }^{*} p<0.01$.

inflammation in vitro (Figure 5A). Mesenteric lymph node cells of mice were cultured in vitro and treated with $L$. reuteri or L. reuteri + LPS to assess the expression of inflammatory cytokines. Inflammatory cytokine expression was increased after LPS stimulation, but the expression of inflammatory cytokines in the MLN was not upregulated in the group coincubated with L. reuteri (Figures 5B-D). These results suggest that $L$. reuteri inhibits inflammatory reactions in vitro to some extent.

\section{Lactobacillus reuteri Attenuates Rituximab-Related Inflammatory Damage}

It has been reported that $L$. reuteri reduces inflammatory reactions and relieves the development of colitis
(Madsen et al., 1999; Liu et al., 2012; Mu et al., 2017, 2018). To verify whether the loss of intestinal $L$. reuteri leads to more severe inflammatory damage, colonic colonization was performed in antibiotic-treated mice with RTX by oral administration of L. reuteri (Figure 6A). After antibiotic treatment (day 0), levels of L. reuteri in the $\mathrm{ATB}, \mathrm{ATB}+\mathrm{RTX}$, and $\mathrm{ATB}+\mathrm{RTX}+$ L. reuteri groups were significantly decreased compared to those in the other three groups (Figure 6B). On day 5 after intragastric administration of $L$. reuteri, the $L$. reuteri content between the $\mathrm{RTX}+L$. reuteri and $\mathrm{ATB}+\mathrm{RTX}+L$. reuteri groups was significantly higher than in the ATB, RTX, and ATB + RTX groups (Figure 6C). These results indicate that L. reuteri has a strong intestinal colonization effect in mice. Consistent with previous reports that $L$. reuteri loss exacerbates colitis, mice treated with RTX for 7 days developed more severe intestinal 
A

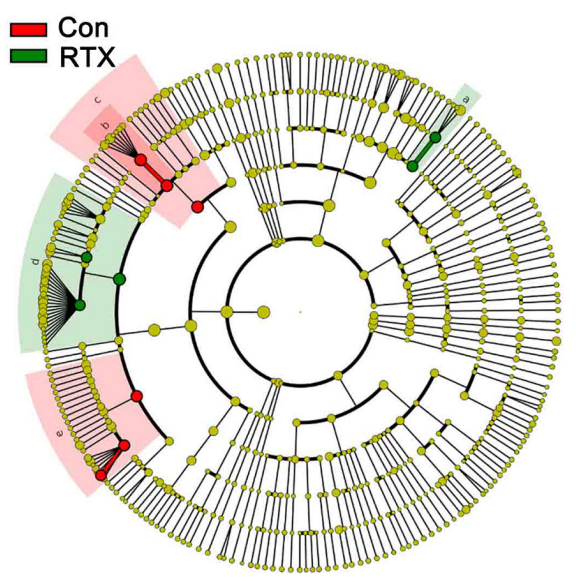

C

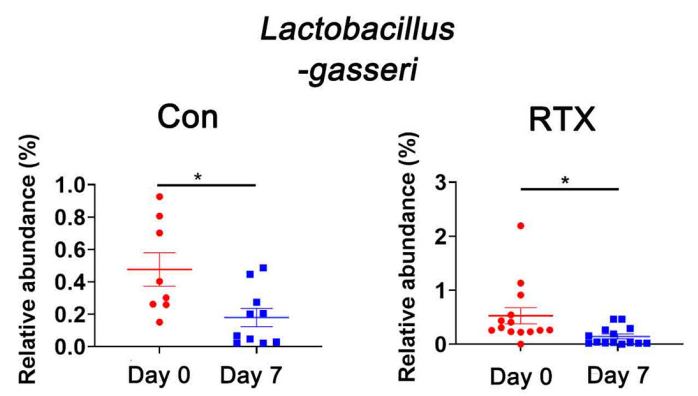

B

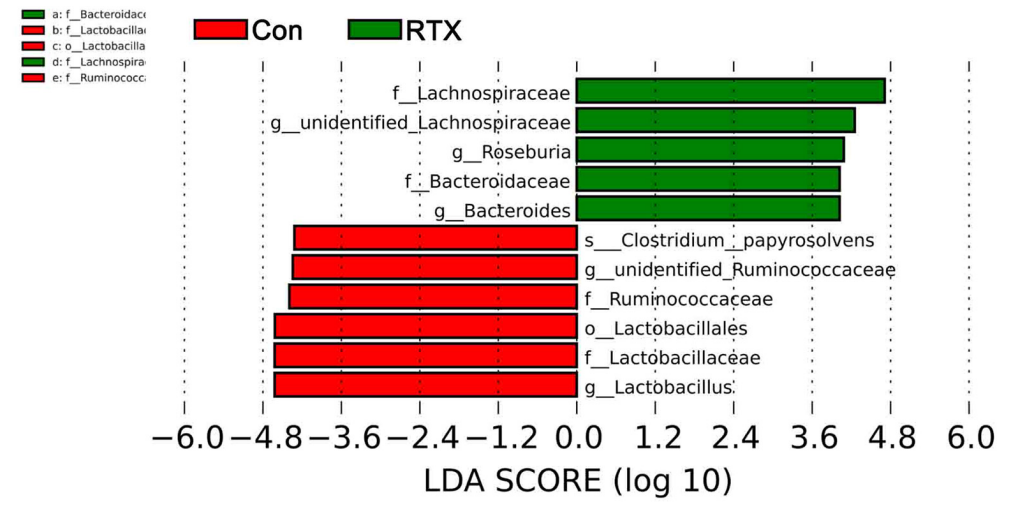

D

\section{Lactobacillus \\ -gasseri}

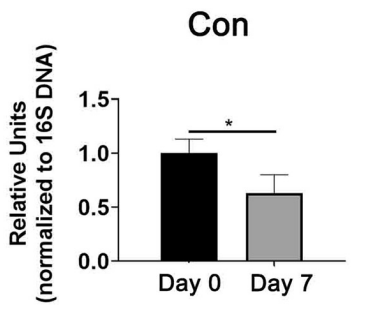

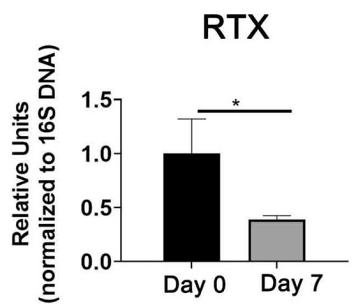

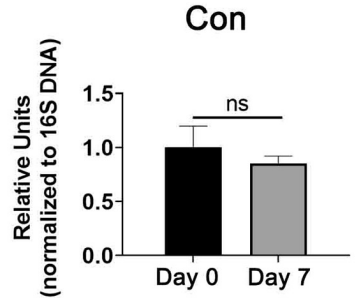

\section{Lactobacillus \\ -reuteri}
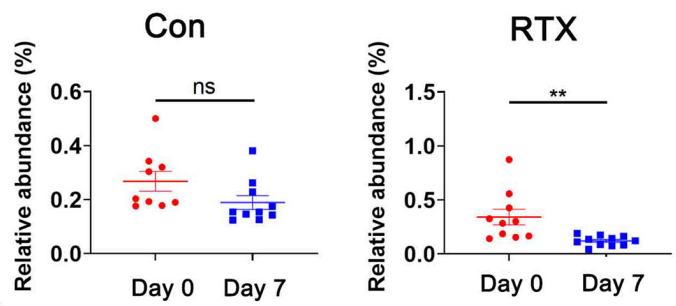

FIGURE 4 | Lactobacillus reuteri is associated with RTX-induced intestinal mucositis. (A) LEfSe analysis of differentially abundant species in RTX-treated mice on day $0(R 1)$ and day $7(R 2)$. (B) LDA scores of differentially abundant species in the intestine before and after RTX treatment showing only the groups with an LDA threshold > 3.6. (C) The relative abundance of significantly different bacteria in control and RTX treatment groups. (D) qPCR results of different bacteria in feces samples. Data are shown as the mean \pm SEM. ${ }^{*} p<0.05,{ }^{* *} p<0.01$.

mucositis, in contrast to the control group, villous atrophy and intestinal epithelial injury of mice in the RTX treatment group were obvious, as shown in Figure 6D. Moreover, obvious inflammatory cell infiltration was apparent in the ileum tissue of ATB and RTX-treated mice (Figure 6D) as assessed by histological analysis of increased epithelial damage (Figure 6D) and shortened intestinal villi of ileum (Figure 6E), which resulted in a reduced mucositis score (Supplementary Figure 2) compared with the RTX-treated control group (Figure 6E). In addition, compared with RTX-treated mice, L. reuteri-colonized mice exhibited decreased levels of inflammatory cytokines (Figure 6F) and increased levels of intestinal tight junction proteins (Figure 6F). These findings suggest that $L$. reuteri alleviates RTX-induced intestinal mucositis and reduces damage to the intestinal mucosal barrier system. Next, we analysed changes in the immune cell population 7 days after L. reuteri was implanted. Compared with the control group, the number of Th1 and Th17 cells in the MLN of RTX-treated mice was significantly 


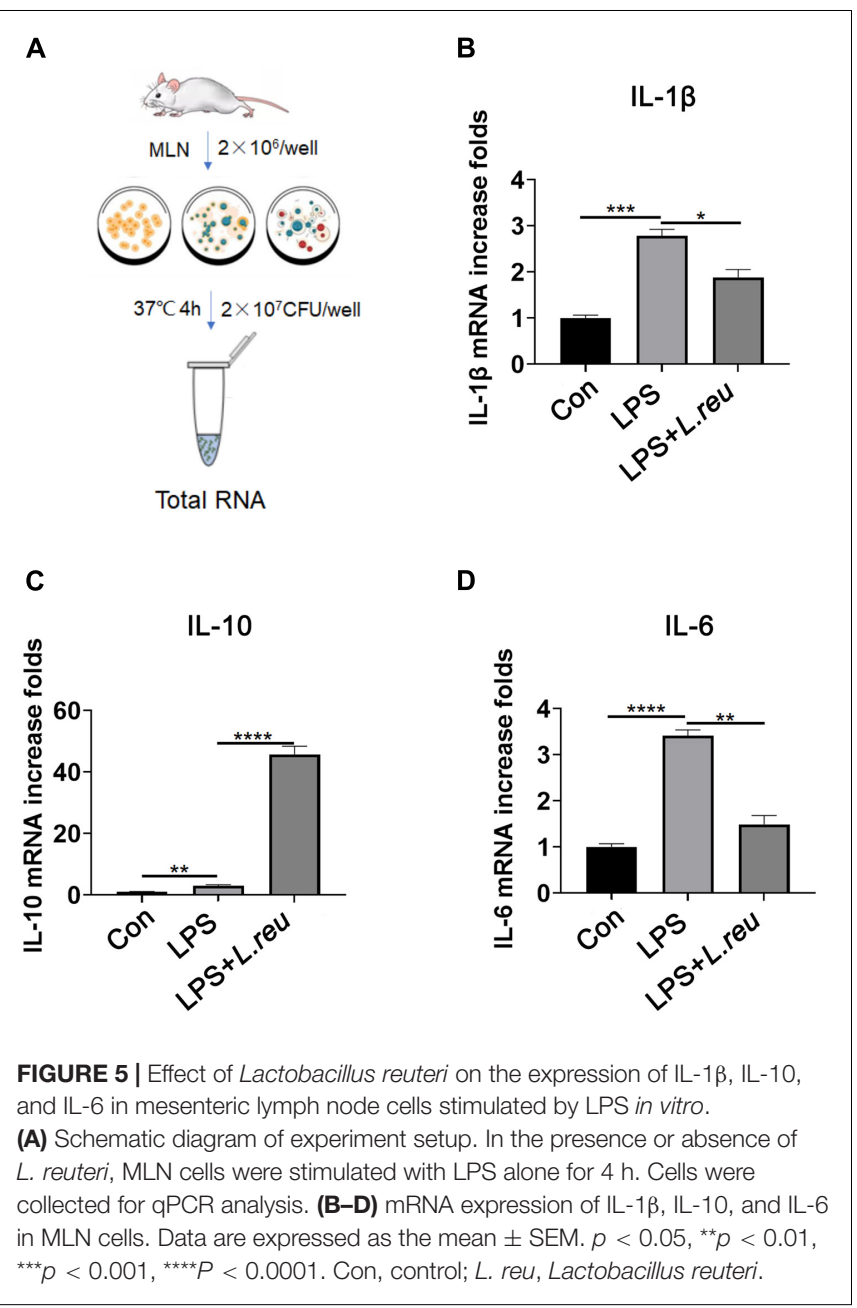

increased (Figure 7A). Consistent with our previous results, RTX treatment resulted in a significant increase in Th1 and Th17 cells in the MLN (Figures 7A,B). However, the number of Th1 and Th17 cells in the MLN of mice fed $L$. reuteri was significantly reduced, and Th2 cells was increased (Supplementary Figure 5). In addition, RTX treatment resulted in a decrease in Breg cells, and intragastric administration of $L$. reuteri resulted in an increase in Treg cells (Supplementary Figure 5) Breg cells in the MLN (Figure 7C). These results suggest that $L$. reuteri could alleviate the intestinal inflammation induced by RTX through regulating the amount of Th1 and Th17 cells.

\section{DISCUSSION}

The toxicity of many anticancer drugs seriously affects their therapeutic effects and the quality of life in cancer patients (El Fassi et al., 2008; Agathocleous et al., 2010; Ardelean et al., 2010; Blombery et al., 2011; Sekkach et al., 2011; Lipka et al., 2016; Ziętarska et al., 2017; Maestá et al., 2018; Shahmohammadi et al., 2018; Kaegi et al., 2019). Some drug treatments lead to intestinal mucosal dysfunction, including endothelial and epithelial cell death and even mucosal immune system activation (ChaveliLópez and Bagán-Sebastián, 2016; de Melo Manzi et al., 2016; Shimamura et al., 2018). At present, there is no successful method to treat mucositis. In recent years, studies have shown that the intestinal microbiota regulates the therapeutic effect of some anticancer drugs (Authors et al., 2015; Sivan et al., 2015; Routy et al., 2018), but little is known about the regulatory function of the intestinal flora in the gastrointestinal toxicity of these drugs (Zhou et al., 2018; Yu et al., 2019). The intestinal mucosa acts as a selective barrier between the body and the external environment that blocks harmful substances from entering the systemic circulation but also ensures the absorption of nutrients (Isaacs-Ten et al., 2020). The intestinal microbiota lives on the layer of mucus secreted by intestinal goblet cells. The intestinal microbiota is involved in the regulation of mucosal barrier function, immune balance, prevention of pathogen infection, vitamin synthesis and metabolism (Gibson, 2009).

Our results suggest that the decrease in $L$. reuteri may be related to RTX-induced mucositis. Previous studies have shown that the absence of $L$. reuteri leads to colic in infants, L. reuteri supplementation alleviates the damage of DSS-induced colitis in mice, and $L$. reuteri can be used to treat enteritis in IL-10-deficient mice (Madsen et al., 1999; Liu et al., 2012; Bene et al., 2017; Mu et al., 2017, 2018; Savino et al., 2018). Other studies have shown that $L$. reuteri may help to alleviate chronic inflammation (Madsen et al., 1999; Liu et al., 2012; Mu et al., 2017, 2018). All these studies have illustrated a close relationship between L. reuteri and inflammation.

We used antibiotics to reduce the abundance of $L$. reuteri in the intestine of mice. We found that RTX induced intestinal mucosal injury and aggravated inflammatory reactions, with marked inflammatory cell infiltration. However, our combined antibiotics were broad-spectrum antibiotics that are mainly used for anaerobic bacteria that not only remove $L$. reuteri but also reduce the content of many intestinal microbiota (Routy et al., 2018; Zhou et al., 2018; Yu et al., 2019), which does not exclude the role of these microbiota in the process of RTX-induced mucositis. Therefore, we designed an additional experimental control group to study the effect of $L$. reuteri on mucositis. The results revealed that $L$. reuteri supplementation alleviates intestinal tissue injury and the inflammatory response induced by RTX. Detection of Claudin-1 in intestinal tissue showed that the damaged intestinal mucosal barrier exhibited signs of repair. These results suggest that L. reuteri alleviates RTXinduced intestinal mucositis. However, the intestinal microbial community is a complex system, and its stability is closely related to the normal digestion, metabolism and immune regulation of the host. The occurrence of intestinal mucositis is not limited to the influence of a single strain but also involves the network regulation mechanism of the formation of other strains or metabolites. The relationship between other strains and RTX-induced intestinal mucositis needs further study. Previous studies have described the mechanism by which the intestinal microbiota regulates the efficacy of anticancer drugs, which can be summarized as the framework of transport, immune regulation, metabolism, enzyme degradation, and diversity reduction (Alexander et al., 2017; Roy and Trinchieri, 2017). 
A

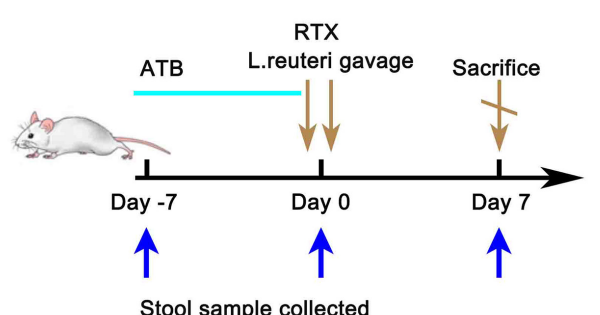

C

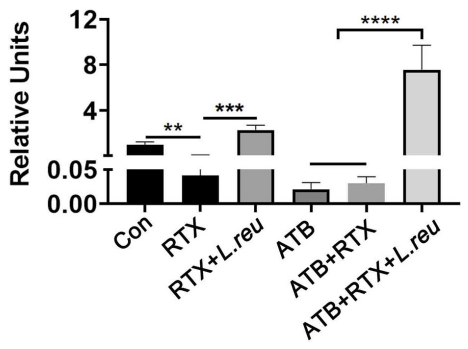

B

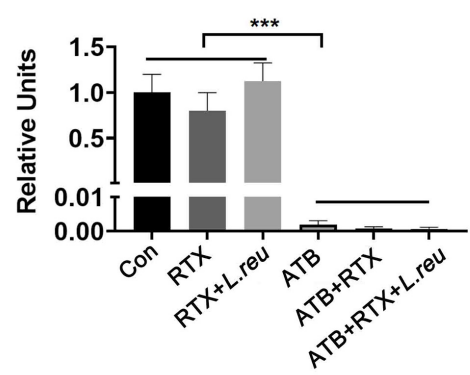

E

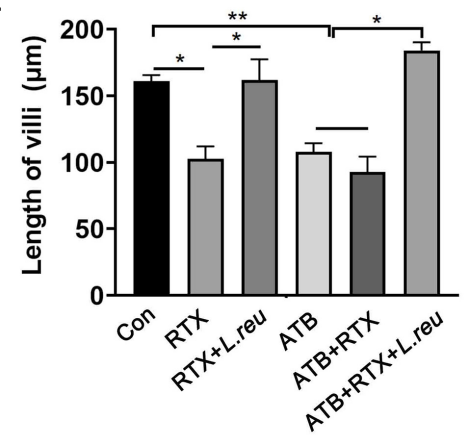

D

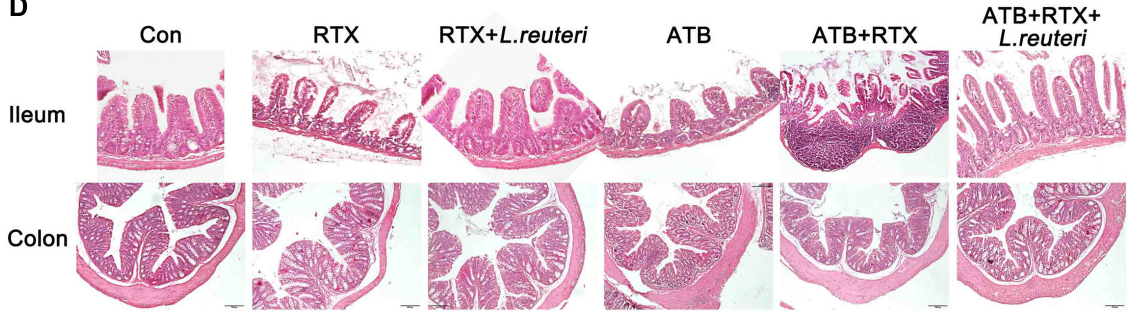

F
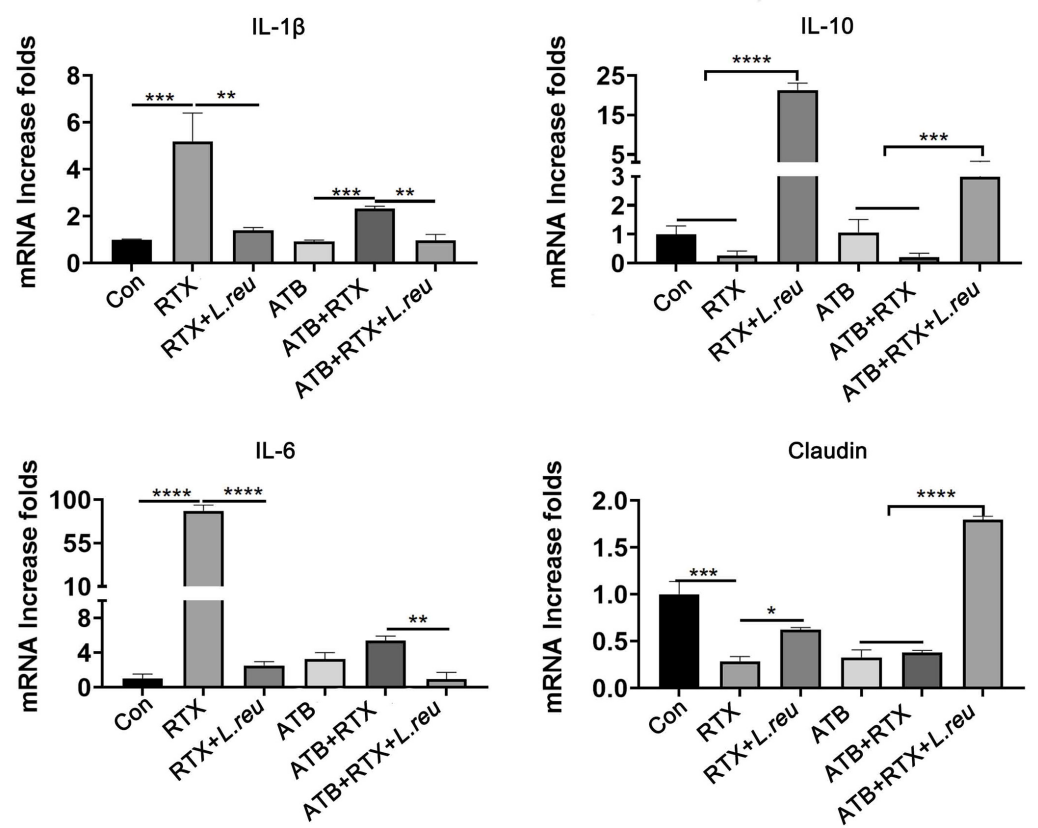

FIGURE 6 | Lactobacillus reuteri attenuates RTX-induced inflammatory injury. (A) Experimental schedule. (B) Relative abundance of $L$. reuteri in the intestine of mice detected by qPCR after 5 days of antibiotic administration. (C) Relative abundance of $L$. reuteri in the intestine detected by qPCR after 5 days of $L$. reuteri gavage. (D) H\&E staining of the ileum and colon (magnification, 40x). (E) Villus length of the ileum. (F) mRNA expression levels of IL-1 $\beta$, IL-6, IL-10, and claudin in the ileum of mice. Data are expressed as the mean \pm SEM. $p<0.05,{ }^{\star \star} p<0.01,{ }^{\star \star \star} p<0.001,{ }^{\star \star \star \star} P<0.0001$. Con, control; ATB, antibiotic treatment; L. reu, L. reuteri. 
A
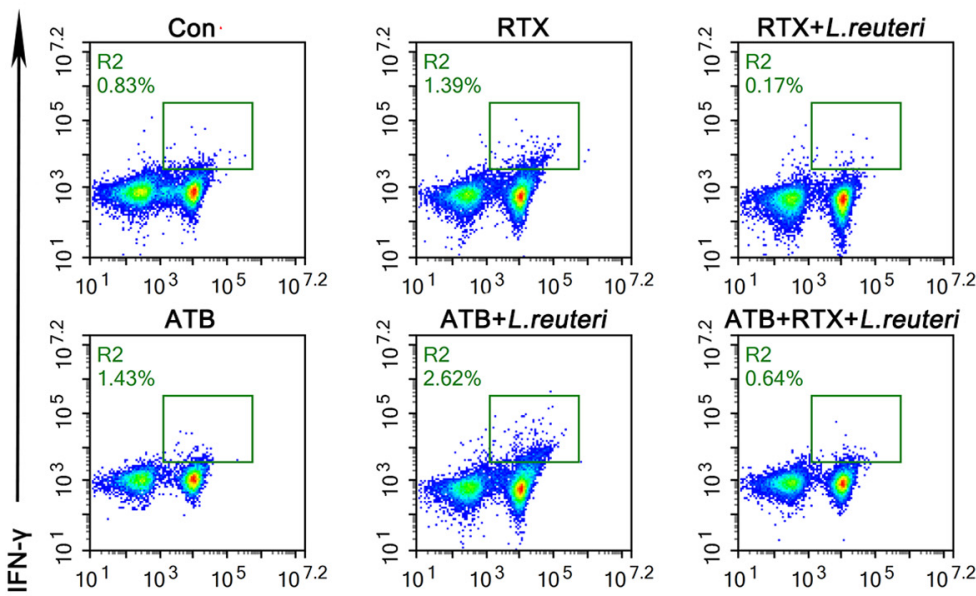

CD4

B
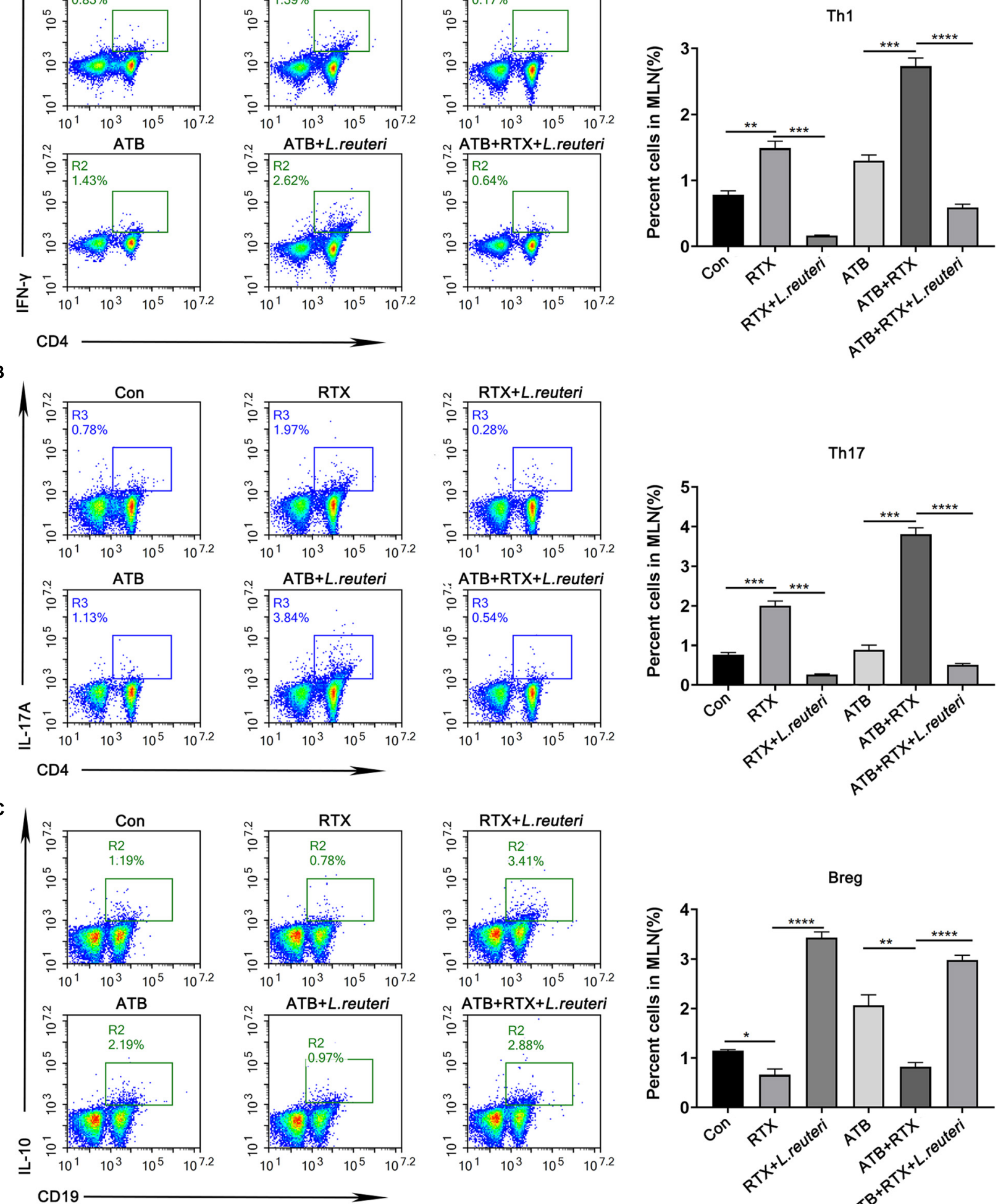

Th17
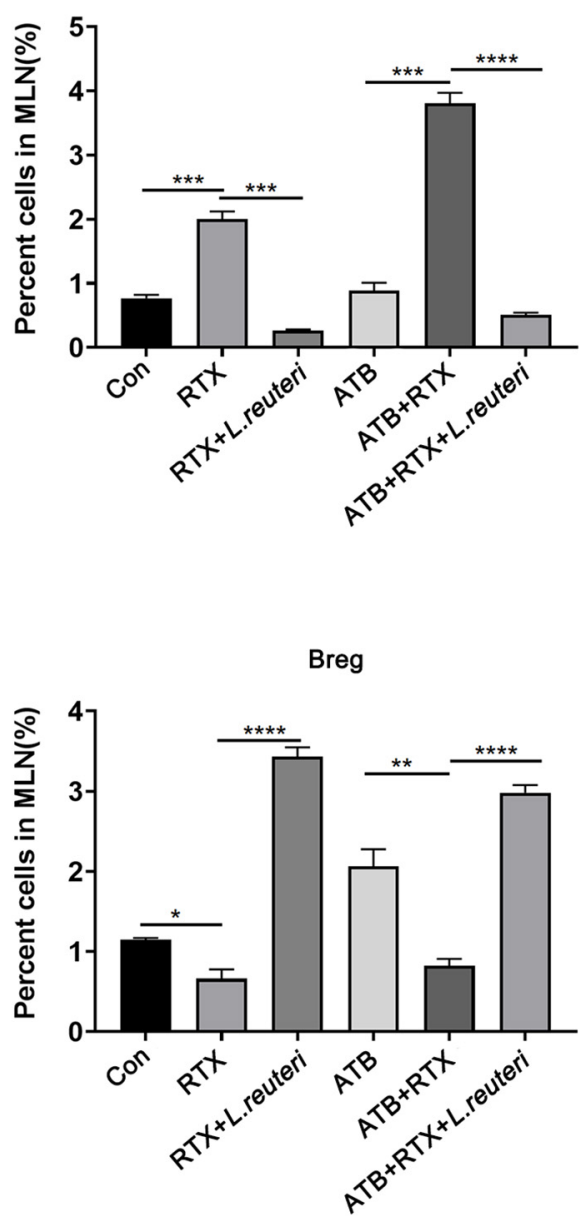

FIGURE 7 | Lactobacillus reuteri supplementation alleviates Th cell imbalance induced by RTX and inhibits Th1 and Th17 immune responses. (A) Th1 cells, (B) Th17 cells, and (C) Breg cells in MLN cells. Data are expressed as the mean \pm SEM. $p<0.05,{ }^{* \star} p<0.01,{ }^{* \star *} p<0.001,{ }^{* \star * \star} P<0.0001$. 
A previous study reported that chemotherapy drugs lead to the destruction of intestinal structure, accompanied by the transfer of some symbiotic bacteria to the secondary lymphoid organs of mice, stimulating the response of Th1 and Th17 cells, and the antitumor effect of the drug was weakened after antibiotic treatment (Viaud et al., 2013; Yu et al., 2019). These data suggest that intestinal microbial translocation caused by intestinal mucosal damage induces an immune response and affects the efficacy of drugs. In this study, RTX caused intestinal atrophy, infiltration of inflammatory cells and destruction of the intestinal mucosal barrier. Therefore, we speculate that RTXinduced intestinal damage may lead to an imbalance in the intestinal microbiota and trigger an immune response. Results demonstrated that Th1 and Th17 cells in MLNs were significantly increased in response to RTX treatment. However, after L. reuteri supplementation, Th cells decreased while Breg cells increased in MLNs. Therefore, L. reuteri may alleviate the gastrointestinal toxicity induced by RTX through Th cells.

Regulatory B cells (Bregs) is a kind of immunosuppressive cells, which can inhibit a variety of immunopathology by producing cytokines such as interleukin-10 (IL-10). Luu et al.'s (2019) research shows that the anti-inflammatory effect of SCFA (especially pentanoate) was not only reflected in strong upregulation of IL-10 production in Bregs but also by a potent suppression of pathogenic Th17 cell phenotype. Yan et al. showed that $L$. reuteri produce short chain fatty acids (SCFAs) as a major fertilization product (Stewart et al., 2009; Den Besten et al., 2013; Furusawa et al., 2013). In addition, Nagpal et al. (2018) and others believe that the SC FA content in the intestine will increase after $L$. reuteri supplementation. It is speculated that L. reuteri may affect the content of Bregs by regulating the metabolism of SCFA. However, the specific mechanism still needs to be further explored.

We demonstrated that $L$. reuteri alleviates the upregulation of inflammatory cytokines in MLN cells stimulated by LPS in vitro. Therefore, L. reuteri alleviates the inflammatory stimulation caused by RTX and inhibits the local and systemic immune response caused by intestinal mucosal damage in response to RTX. Although this study shows some promising results, it also has limitations. RTX is an anticancer drug widely used in nonHodgkin's lymphoma. It is also related to changes in the intestinal flora. We should conduct more experiments in cancer models not limited to normal mice to verify the relationship between the gut

\section{REFERENCES}

Acharya, N., Madi, A., Zhang, H., Klapholz, M., Escobar, G., Dulberg, S., et al. (2020). Endogenous glucocorticoid signaling regulates CD8+ $\mathrm{T}$ cell differentiation and development of dysfunction in the tumor microenvironment. Immunity 53, 658-671.e6. doi: 10.1016/j.immuni.2020. 08.005

Agathocleous, A., Rohatiner, A., Rule, S., Hunter, H., Kerr, J. P., Neeson, S. M., et al. (2010). Weekly versus twice weekly bortezomib given in conjunction with rituximab, in patients with recurrent follicular lymphoma, mantle cell lymphoma and Waldenström macroglobulinaemia. Br. J. Haematol. 51, 346353. doi: 10.1111/j.1365-2141.2010.08340.x

Alexander, J. L., Wilson, I. D., Teare, J., Marchesi, J. R., Nicholson, J. K., and Kinross, J. M. (2017). Gut microbiota modulation of chemotherapy efficacy and microbiota and RTX treatment. In conclusion, our data suggest that the regulation of intestinal microbiota may be a new way to reduce the toxicity of anticancer drugs.

\section{DATA AVAILABILITY STATEMENT}

The datasets presented in this study can be found in online repositories. The names of the repository/repositories and accession number(s) can be found in the article/ Supplementary Material.

\section{ETHICS STATEMENT}

The animal study was reviewed and approved by the Animal Protection Committee of Sichuan University (Sichuan, China).

\section{AUTHOR CONTRIBUTIONS}

LY, BaZ, and BiZ contributed to conception and design of the study. BaZ organized the database. CD performed the statistical analysis. BiZ wrote the first draft of the manuscript. RZ, DX, and YT wrote sections of the manuscript. All authors contributed to manuscript revision, read, and approved the submitted version.

\section{FUNDING}

This study was supported by grants from the National Science and Technology Major Project (Nos. 2018ZX09201018-013 and 2018ZX09733001-001-007), "Leading Academic of Tenthousand Talents Program" and 1.3.5 project for disciplines of excellence, West China Hospital, Sichuan University. All the funding from these sources were used for data collection and analysis.

\section{SUPPLEMENTARY MATERIAL}

The Supplementary Material for this article can be found online at: https://www.frontiersin.org/articles/10.3389/fmicb. 2021.645500/full\#supplementary-material

toxicity. Nat. Rev. Gastroenterol. Hepatol. 14, 356-365. doi: 10.1038/nrgastro. 2017.20

Ardelean, D. S., Gonska, T., Wires, S., Cutz, E., Griffiths, A., Harvey, E., et al. (2010). Severe ulcerative colitis after rituximab therapy. Pediatrics 126, e243-e246. doi: 10.1542/peds.2009-3395

Authors, F., Benyamin, F. W., Man Lei, Y., Jabri, B., Alegre, M.-L., Chang, E. B., et al. (2015). Anticancer immunotherapy by CTLA-4 blockade relies on the gut microbiota. Science 350, 1079-1084.

Bau, J., Alhassan, B., Roth, J., Nguyen, J., Harris, X., Buie, C., et al. (2016). Mo1922 Keratinocyte growth factor protects against $C$. difficile toxin-induced injury, cell death and apopotisis in vitro and in vivo. Gastroenterology 150, S816-S817. doi: 10.1016/s0016-5085(16)32764-0

Bene, K. P., Kavanaugh, D. W., Leclaire, C., Gunning, A. P., MacKenzie, D. A., Wittmann, A., et al. (2017). Lactobacillus reuteri surface mucus adhesins 
upregulate inflammatory responses through interactions with innate C-Type lectin receptors. Front. Microbiol. 8:321. doi: 10.3389/fmicb.2017.00321

Beutheu, S., Ouelaa, W., Guérin, C., Belmonte, L., Aziz, M., Tennoune, N., et al. (2014). Glutamine supplementation, but not combined glutamine and arginine supplementation, improves gut barrier function during chemotherapy-induced intestinal mucositis in rats. Clin. Nutr. 33, 694-701. doi: 10.1016/j.clnu.2013. 09.003

Blombery, P., Prince, H. M., Levinson, M., Pianko, S., Maxwell, E., and Bhathal, P. (2011). Rituximab-induced immunodysregulatory ileocolitis in a patient with follicular lymphoma. J. Clin. Oncol. 29, e110-e112. doi: 10.1200/JCO.2010.31. 8899

Bokulich, N. A., Subramanian, S., Faith, J. J., Gevers, D., Gordon, J. I., Knight, R., et al. (2013). Quality-filtering vastly improves diversity estimates from Illumina amplicon sequencing. Nat. Methods 10, 57-59. doi: 10.1038/nmeth.2276

Boukhettala, N., Leblond, J., Claeyssens, S., Faure, M., Le Pessot, F., Bôle-Feysot, C., et al. (2009). Methotrexate induces intestinal mucositis and alters gut protein metabolism independently of reduced food intake. Am. J. Physiol. Endocrinol. Metab. 296, E182-E190. doi: 10.1152/ajpendo.90459.2008

Bullman, S., Pedamallu, C. S., Sicinska, E., Clancy, T. E., Zhang, X., Cai, D., et al. (2017). Analysis of Fusobacterium persistence and antibiotic response in colorectal cancer. Science 358, 1443-1448. doi: 10.1126/science.aal5240

Carrasco, A., and Fernández-Bañares, F. (2017). Th1 pathway: the missing link between inflammatory bowel disease and microscopic colitis? Dig. Dis. Sci. 62, 2609-2611. doi: 10.1007/s10620-017-4692-x

Chae, B. S. (2018). Pretreatment of low-dose and super-low-dose LPS on the production of in vitro LPS-induced inflammatory mediators. Toxicol. Res. 34, 65-73. doi: 10.5487/TR.2018.34.1.065

Chaveli-López, B., and Bagán-Sebastián, J. V. (2016). Treatment of oral mucositis due to chemotherapy. J. Clin. Exp. Dent. 8, e201-e209. doi: 10.4317/jced.52917

de Melo Manzi, N., Silveira, R. C., and dos Reis, P. E. D. (2016). Prophylaxis for mucositis induced by ambulatory chemotherapy: systematic review. J. Adv. Nurs. 72, 735-746. doi: 10.1111/jan.12867

Den Besten, G., Van Eunen, K., Groen, A. K., Venema, K., Reijngoud, D. J., and Bakker, B. M. (2013). The role of short-chain fatty acids in the interplay between diet, gut microbiota, and host energy metabolism. J. Lipid Res. 54, 2325-2340. doi: 10.1194/jlr.R036012

Edgar, R. C. (2004). MUSCLE: multiple sequence alignment with high accuracy and high throughput. Nucleic Acids Res. 32, 1792-1797. doi: 10.1093/nar/gkh340

Edgar, R. C. (2013). UPARSE: highly accurate OTU sequences from microbial amplicon reads. Nat. Methods 10, 996-998. doi: 10.1038/nmeth. 2604

Edgar, R. C., Haas, B. J., Clemente, J. C., Quince, C., and Knight, R. (2011). UCHIME improves sensitivity and speed of chimera detection. Bioinformatics 27, 2194-2200. doi: 10.1093/bioinformatics/btr381

El Fassi, D., Nielsen, C. H., Kjeldsen, J., Clemmensen, O., and Hegedüs, L. (2008). Ulcerative colitis following B lymphocyte depletion with rituximab in a patient with Graves' disease. Gut 57, 714-715. doi: 10.1136/gut.2007.138305

Farrell, C. L., Bready, J. V., Rex, K. L., Chen, J. N., DiPalma, C. R., Whitcomb, K. L., et al. (1998). Keratinocyte growth factor protects mice from chemotherapy and radiation-induced gastrointestinal injury and mortality. Cancer Res. 8, 933-939.

Furusawa, Y., Obata, Y., Fukuda, S., Endo, T. A., Nakato, G., Takahashi, D., et al. (2013). Commensal microbe-derived butyrate induces the differentiation of colonic regulatory T cells. Nature 504, 446-450. doi: 10.1038/nature12721

Gibson, R. J. (2009). Gut microbiome and intestinal mucositis: a new challenge for researchers. Cancer Biol. Ther. 8, 512-513. doi: 10.4161/cbt.8.6.7852

Gopalakrishnan, V., Spencer, C. N., Nezi, L., Reuben, A., Andrews, M. C., Karpinets, T. V., et al. (2018). Gut microbiome modulates response to antiPD-1 immunotherapy in melanoma patients. Science 359, 97-103. doi: 10.1126/ science.aan 4236

Haas, B. J., Gevers, D., Earl, A. M., Feldgarden, M., Ward, D. V., Giannoukos, G., et al. (2011). Chimeric $16 \mathrm{~S}$ rRNA sequence formation and detection in Sanger and 454-pyrosequenced PCR amplicons. Genome Res. 21, 494-504. doi: 10.1101/gr.112730.110

Isaacs-Ten, A., Echeandia, M., Moreno-Gonzalez, M., Brion, A., Goldson, A., Philo, M., et al. (2020). Intestinal microbiome-macrophage crosstalk contributes to cholestatic liver disease by promoting intestinal permeability. Hepatology 72 , 2090-2108. doi: 10.1002/hep.31228
Jiménez-Flores, R., Brisson, G., Payken, H. F., and Sharpe, J. P. (2010). Characterization of lactobacillus reuteri interaction with milk fat globule membrane components in dairy products. J. Agric. Food Chem. 58, 5612-5619. doi: $10.1021 /$ jf904381s

Kaegi, C., Wuest, B., Schreiner, J., Steiner, U. C., Vultaggio, A., Matucci, A., et al. (2019). Systematic review of safety and efficacy of rituximab in treating immune-mediated disorders. Front. Immunol. 10:1990. doi: 10.3389/fimmu. 2019.01990

Kumawat, A. K., Strid, H., Tysk, C., Bohr, J., and Hörnquist, E. H. (2013). Microscopic colitis patients demonstrate a mixed Th17/Tc17 and Th1/Tc1 mucosal cytokine profile. Mol. Immunol. 55, 355-364. doi: 10.1016/j.molimm. 2013.03.007

Li, H., He, J., and Jia, W. (2016). The influence of gut microbiota on drug metabolism and toxicity. Expert Opin. Drug Metab. Toxicol. 12, 31-40. doi: $10.1517 / 17425255.2016 .1121234$

Li, H. L., Lu, L., Wang, X. S., Qin, L. Y., Wang, P., Qiu, S. P., et al. (2017). Alteration of gut microbiota and inflammatory cytokine/chemokine profiles in 5-fluorouracil induced intestinal mucositis. Front. Cell. Infect. Microbiol. 7:455. doi: 10.3389/fcimb.2017.00455

Lipka, S., Katz, S., and Crawford, J. M. (2016). Fulminant colitis following rituximab therapy. Gastroenterol. Hepatol. 12, 58-60.

Liu, Y., Fatheree, N. Y., Mangalat, N., and Rhoads, J. M. (2012). Lactobacillus reuteri strains reduce incidence and severity of experimental necrotizing enterocolitis via modulation of TLR4 and NF-KB signaling in the intestine. Am. J. Physiol. Gastrointest. Liver Physiol. 302, G608-G617. doi: 10.1152/ajpgi.00266. 2011

Luu, M., Pautz, S., Kohl, V., Singh, R., Romero, R., Lucas, S., et al. (2019). The short-chain fatty acid pentanoate suppresses autoimmunity by modulating the metabolic-epigenetic crosstalk in lymphocytes. Nat. Commun. 10:760. doi: 10. 1038/s41467-019-08711-2

Madsen, K. L., Doyle, J. S., Jewell, L. D., Tavernini, M. M., and Fedorak, R. N. (1999). Lactobacillus species prevents colitis in interleukin 10 gene-deficient mice. Gastroenterology 116, 1107-1114. doi: 10.1016/S0016-5085(99)70013-2

Maestá, I., Nitecki, R., Horowitz, N. S., Goldstein, D. P., de Freitas Segalla Moreira, M., Elias, K. M., et al. (2018). Effectiveness and toxicity of first-line methotrexate chemotherapy in low-risk postmolar gestational trophoblastic neoplasia: the New England trophoblastic disease center experience. Gynecol. Oncol. 148, 161-167. doi: 10.1016/j.ygyno.2017.10.028

Magoč, T., and Salzberg, S. L. (2011). FLASH: fast length adjustment of short reads to improve genome assemblies. Bioinformatics 27, 2957-2963. doi: 10.1093/ bioinformatics/btr507

Mantovani, A., Sozzani, S., Locati, M., Allavena, P., and Sica, A. (2002). Macrophage polarization: tumor-associated macrophages as a paradigm for polarized M2 mononuclear phagocytes. Trends Immunol. 23, 549-555. doi: 10.1016/S1471-4906(02)02302-5

Martinez, F. O., and Gordon, S. (2014). The M1 and M2 paradigm of macrophage activation: time for reassessment. F1000Prime Rep. 6:13. doi: 10.12703/P6-13

Matson, V., Fessler, J., Bao, R., Chongsuwat, T., Zha, Y., Alegre, M. L., et al. (2018). The commensal microbiome is associated with anti-PD-1 efficacy in metastatic melanoma patients. Science 359, 104-108. doi: 10.1126/science.aao3290

$\mathrm{Mu}$, Q., Tavella, V. J., and Luo, X. M. (2018). Role of Lactobacillus reuteri in human health and diseases. Front. Microbiol. 9:757. doi: 10.3389/fmicb.2018.00757

Mu, Q., Zhang, H., Liao, X., Lin, K., Liu, H., Edwards, M. R., et al. (2017). Control of lupus nephritis by changes of gut microbiota. Microbiome 5:73. doi: 10.1186/s40168-017-0300-8

Nagpal, R., Wang, S., Ahmadi, S., Hayes, J., Gagliano, J., Subashchandrabose, S., et al. (2018). Human-origin probiotic cocktail increases short-chain fatty acid production via modulation of mice and human gut microbiome. Sci. Rep. 8:12649. doi: 10.1038/s41598-018-30114-4

Quast, C., Pruesse, E., Yilmaz, P., Gerken, J., Schweer, T., Yarza, P., et al. (2013). The SILVA ribosomal RNA gene database project: improved data processing and web-based tools. Nucleic Acids Res. 41, D590-D596. doi: 10.1093/nar/gks1219

Rauber, S., Luber, M., Weber, S., Maul, L., Soare, A., Wohlfahrt, T., et al. (2017). Resolution of inflammation by interleukin-9-producing type 2 innate lymphoid cells. Nat. Med. 23, 938-944. doi: 10.1038/nm.4373

Rothhammer, V., Mascanfroni, I. D., Bunse, L., Takenaka, M. C., Kenison, J. E., Mayo, L., et al. (2016). Type i interferons and microbial metabolites of tryptophan modulate astrocyte activity and central nervous system 
inflammation via the aryl hydrocarbon receptor. Nat. Med. 22, 586-597. doi: 10.1038/nm.4106

Routy, B., Le Chatelier, E., Derosa, L., Duong, C. P. M., Alou, M. T., Daillère, R., et al. (2018). Gut microbiome influences efficacy of PD-1-based immunotherapy against epithelial tumors. Science 359, 91-97. doi: 10.1126/ science.aan 3706

Roy, S., and Trinchieri, G. (2017). Microbiota: a key orchestrator of cancer therapy. Nat. Rev. Cancer 17, 271-285. doi: 10.1038/nrc.2017.13

Savino, F., Galliano, I., Garro, M., Savino, A., Daprà, V., Montanari, P., et al. (2018). Regulatory T cells and Toll-like receptor 2 and 4 mRNA expression in infants with colic treated with Lactobacillus reuteri DSM17938. Benef. Microbes 9, 917-925. doi: 10.3920/BM2017.0194

Segata, N., Izard, J., Waldron, L., Gevers, D., Miropolsky, L., Garrett, W. S., et al. (2011). Metagenomic biomarker discovery and explanation. Genome Biol. 12:R60. doi: 10.1186/gb-2011-12-6-r60

Sekkach, Y., Hammi, S., Elqatni, M., Fatihi, J., Badaoui, M., Elomri, N., et al. (2011). La colite ulcérative: une conséquence exceptionnelle après traitement par rituximab. Ann. Pharm. Fr. 69, 265-269. doi: 10.1016/j.pharma.2011. 06.005

Sender, R., Fuchs, S., and Milo, R. (2016). Revised estimates for the number of human and bacteria cells in the body. PLoS Biol. 14:e1002533. doi: 10.1371/ journal.pbio. 1002533

Shahmohammadi, S., Sahraian, M. A., Shahmohammadi, A., Doosti, R., ZareMirzaie, A., and Naser Moghadasi, A. (2018). A presentation of ulcerative colitis after rituximab therapy in a patient with multiple sclerosis and literature review. Mult. Scler. Relat. Disord. 22, 22-26. doi: 10.1016/j.msard.2018. 02.030

Shimamura, Y., Takeuchi, I., Terada, H., and Makino, K. (2018). A mouse model for oral mucositis induced by cancer chemotherapy. Anticancer Res. 38, 307-312. doi: 10.21873 /anticanres. 12223

Sica, A., and Bronte, V. (2007). Altered macrophage differentiation and immune dysfunction in tumor development. J. Clin. Invest. 117, 1155-1166. doi: 10.1172/ JCI31422

Sica, A., and Mantovani, A. (2012). Macrophage plasticity and polarization: in vivo veritas. J. Clin. Invest. 122, 787-795. doi: 10.1172/JCI59643

Sivan, A., Corrales, L., Hubert, N., Williams, J. B., Aquino-Michaels, K., Earley, Z. M., et al. (2015). Commensal bifidobacterium promotes antitumor immunity and facilitates anti-PD-L1 efficacy. Science 350, 1084-1089.

Stewart, M. L., Savarino, V., and Slavin, J. L. (2009). Assessment of dietary fiber fermentation: effect of Lactobacillus reuteri and reproducibility of short-chain fatty acid concentrations. Mol. Nutr. Food Res. 53(Suppl. 1), S114-S120. doi: 10.1002/mnfr.200700523

Vétizou, M., Pitt, J. M., Daillère, R., Lepage, P., Waldschmitt, N., Flament, C., et al. (2015). Anticancer immunotherapy by CTLA-4 blockade relies on the gut microbiota. Science 350, 1079-1084. doi: 10.1126/science.aad1329
Viaud, S., Saccheri, F., Mignot, G., Yamazaki, T., Daillère, R., Hannani, D., et al. (2013). The intestinal microbiota modulates the anticancer immune effects of cyclophosphamide. Science 42, 971-976. doi: 10.1126/science.1240537

Wardill, H. R., and Tissing, W. J. E. (2017). Determining risk of severe gastrointestinal toxicity based on pretreatment gut microbial community in patients receiving cancer treatment: a new predictive strategy in the quest for personalized cancer medicine. Curr. Opin. Support. Palliat. Care. 11, 125-132. doi: 10.1097/SPC.0000000000000265

Wilson, I. D., and Nicholson, J. K. (2017). Gut microbiome interactions with drug metabolism, efficacy, and toxicity. Transl. Res. 179, 204-222. doi: 10.1016/j.trsl. 2016.08.002

Yu, C., Zhou, B., Xia, X., Chen, S., Deng, Y., Wang, Y., et al. (2019). Prevotella copri is associated with carboplatin-induced gut toxicity. Cell Death Dis. 10:714. doi: 10.1038/s41419-019-1963-9

Zhou, B., Xia, X., Wang, P., Chen, S., Yu, C., Huang, R., et al. (2018). Induction and amelioration of methotrexate-induced gastrointestinal toxicity are related to immune response and gut microbiota. EBioMedicine 33, 122-133. doi: 10. 1016/j.ebiom.2018.06.029

Ziętarska, M., Krawczyk-Lipiec, J., Kraj, L., Zaucha, R., and Małgorzewicz, S. (2017). Chemotherapy-related toxicity, nutritional status and quality of life in precachectic oncologic patients with, or without, high protein nutritional support. A prospective, randomized study. Nutrients 9:1108. doi: 10.3390/ nu9101108

Zimmermann, M., Zimmermann-Kogadeeva, M., Wegmann, R., and Goodman, A. L. (2019). Separating host and microbiome contributions to drug pharmacokinetics and toxicity. Science 363:eaat9931. doi: 10.1126/science. aat9931

Conflict of Interest: The authors declare that the research was conducted in the absence of any commercial or financial relationships that could be construed as a potential conflict of interest.

Publisher's Note: All claims expressed in this article are solely those of the authors and do not necessarily represent those of their affiliated organizations, or those of the publisher, the editors and the reviewers. Any product that may be evaluated in this article, or claim that may be made by its manufacturer, is not guaranteed or endorsed by the publisher.

Copyright (c) 2021 Zhao, Zhou, Dong, Zhang, Xie, Tian and Yang. This is an openaccess article distributed under the terms of the Creative Commons Attribution License (CC BY). The use, distribution or reproduction in other forums is permitted, provided the original author(s) and the copyright owner(s) are credited and that the original publication in this journal is cited, in accordance with accepted academic practice. No use, distribution or reproduction is permitted which does not comply with these terms. 